\title{
Spectroscopic and molecular modeling studies of binding interaction between bovine serum albumin and roflumilast
}

This article was published in the following Dove Press journal:

Drug Design, Development and Therapy

\author{
Tanveer A Wani' \\ Ahmed H Bakheit ${ }^{1,2}$ \\ Mohammed Nazam Ansari ${ }^{3}$ \\ Abdul-Rahman A Al-Majed' \\ Bakr M Al-Qahtani ${ }^{1,4}$ \\ Seema Zargar ${ }^{5}$ \\ 'Department of Pharmaceutical \\ Chemistry, College of Pharmacy, King \\ Saud University, Riyadh, Saudi Arabia; \\ ${ }^{2}$ Department of Chemistry, Faculty of \\ Science and Technology, Al-Neelain \\ University, Khartoum, Sudan; \\ ${ }^{3}$ Department of Pharmacology, \\ College of Pharmacy, Prince Sattam \\ Bin Abdulaziz University, Al Kharj, \\ Saudi Arabia; ${ }^{4}$ Drug Sector, Saudi \\ Food and Drug Authority, Dammam, \\ Saudi Arabia; ${ }^{5}$ Department of \\ Biochemistry, College of Science, King \\ Saud University, Riyadh, Saudi Arabia
}

Correspondence: Tanveer A Wani Department of Pharmaceutical Chemistry, College of Pharmacy, King Saud University, PO Box 2457, Riyadh I 145I, Saudi Arabia

Email twani@ksu.edu.sa
Background: The binding interaction between bovine serum albumin (BSA) and roflumilast (ROF) was explored in this study. The binding of drugs to albumin plays a vital role in their pharmacokinetics and pharmacodynamics in vivo. The mechanisms involved in the interaction between BSA and ROF was studied using multi-spectroscopic experimental and computational approaches.

Materials and methods: Spectrofluorometric experiments were used to determine the method of quenching involved and the conformational changes in the BSA. UV-visible spectroscopy synchronous and three-dimensional fluorescence spectroscopy were used to further explore the binding interaction mechanism.

Results: The results suggested that the intrinsic fluorescence of BSA was quenched due to the formation of a static complex between ROF and BSA. Conformational changes in BSA were determined based on its interaction with ROF. The thermodynamic results suggested that the interaction between ROF and BSA was spontaneous and a hydrophobic interaction occurred between them. Site I of BSA was suggested as the site of interaction between ROF and BSA based on the site marker experiments.

Conclusion: The molecular simulation results and the experimental outcomes were complimentary to each other and helped to identify the binding site and nature of bonds involved in the interaction between ROF and BSA.

Keywords: roflumilast, bovine serum albumin, COPD, fluorescence, quenching

\section{Introduction}

COPD is a pulmonary disorder that is progressive and causes deterioration in lung function. ${ }^{1}$ Patients with severe COPD along with chronic bronchitis and exacerbations are treated with roflumilast (ROF), a potent and selective PDE4 inhibitor (Figure 1)., Patients with COPD exacerbation have higher levels of inflammatory and cytokine markers than patients without exacerbations. The selective inhibition of PDE4 by ROF causes suppression of these inflammatory and cytokine markers in addition to allergen-induced inflammation. ${ }^{4}$ ROF is also known to suppress lipopolysaccharideinduced systemic inflammation. ${ }^{2}$

A major proportion of serum albumin is comprised of extracellular plasma proteins (approximately 60\%). ${ }^{5}$ Serum albumin binds to various ligands (hormones, fatty acids, drugs etc), thus playing a significant role in the pharmacokinetic behavior of these ligands. ${ }^{6}$ The therapeutic efficiency of the ligands depend on their ability to bind to BSA and the mechanism involved in the binding. ${ }^{7-11}$ Bovine serum albumin is commonly substituted for human serum albumin due to its structural similarity, low procurement 


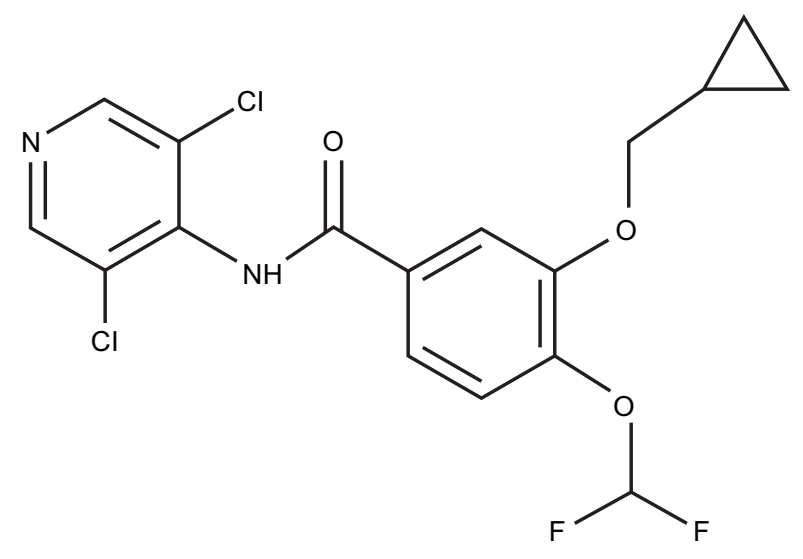

Figure I Chemical structure of ROF.

Abbreviation: ROF, roflumilast.

cost, and availability. The similarity between BSA and human serum albumin is $76 \%$ in terms of identity and $88 \%$ in terms of amino acid sequences. ${ }^{12,13}$ Several studies have explored the interactions between ligands and serum albumin. ${ }^{13-19}$ These binding studies are useful in obtaining information about possible drug-drug interactions, drug: protein resistance, drug side effects, and dosage information. ${ }^{19-21}$

In this study, the mechanism involved in the interaction between ROF and BSA was analyzed. The interaction between ROF and BSA was studied in terms of calculation of binding constants, binding site identification for ROF on BSA, and conformational change in BSA upon interaction with ROF. Ultraviolet-visible (UV-vis) absorption, fluorescence, synchronous fluorescence (SF), and three-dimensional fluorescence spectroscopy studies were conducted to understand the binding interaction. In addition to these spectroscopic studies, molecular simulation studies were also conducted. This study will be useful in understanding the in vivo metabolism, distribution, and pharmacokinetics of ROF.

\section{Materials and methods Materials}

ROF was obtained from Beijing Mesochem Technology Limited (Beijing, China). BSA was obtained from Sisco Research Laboratories (Mumbai, India), and phenylbutazone ( $\geq 99.9 \%$ ) and ibuprofen ( $\geq 99.9 \%$ ) were procured from National Scientific Company (Jeddah, Saudi Arabia). All other materials used including those used to prepare buffers were of analytical grade.

\section{Sample preparation}

ROF was dissolved in dimethyl sulfoxide $(500 \mu \mathrm{L})$ and its stock $\left(1.15 \times 10^{-4} \mathrm{M}\right)$ was prepared in phosphate buffer ( $\mathrm{pH}$ 7.4). The working standards were in the range of $7.46 \times 10^{-7}-1.19 \times 10^{-5} \mathrm{M}$. BSA stock $\left(1.5 \times 10^{-6} \mathrm{M}\right)$ was also prepared in phosphate buffer $(\mathrm{pH} 7.4)$. The sample solutions were prepared using deionized water from Flex Type-IV instrument (Elga Lab Water, Lane End, UK). The stock solutions were stored in refrigerator and fresh working standards were prepared for analysis from the stock solution.

\section{Fluorescence measurements}

The amino acids tryptophan (Trp), tyrosine (Tyr), and phenylalanine (Phe) are fluorescent in nature, thus contributing to the fluorescence of the protein in which they are present. ${ }^{22}$ Among the three amino acid residues, Trp is highly fluorescent and sensitive to the microenvironmental changes that might occur in its vicinity whereas Phe does not get excited (for the most part) easily and has a low quantum yield. Owing to these reasons, Trp is usually used in quenching studies. The two Trp residues found in BSA are Trp-134 and Trp-213, and the intrinsic fluorescence of BSA is dominated by the Trp residues with high quantum yield. Trp-134 is present on BSA surface, whereas Trp-213 is present inside the BSA hydrophobic pocket. A JASCO FP-8200 spectrofluorometer was utilized to measure the fluorescence spectrum. The fluorescence quenching spectra were recorded at $\lambda_{\text {ex }}$ (excitation wavelength) of $280 \mathrm{~nm}$ and $\lambda_{\text {em }}$ (emission wavelength) of 300-500 $\mathrm{nm}$. The fluorescence spectra were recorded at temperatures of 298, 303, and $308 \mathrm{~K}$. BSA spectra were attained in the presence and absence of ROF. A reduction in fluorescence intensity of the sample solution can occur because of inner filter effects and, therefore, needs to be corrected. The effect was corrected as follows:

$$
F_{\text {cor }}=F_{\text {obs }} \times e^{\left(A_{\mathrm{ex}}+A_{\mathrm{em}}\right) / 2}
$$

where $F_{\text {cor }}$ and $F_{\text {obs }}$ represent the corrected and observed fluorescence intensity, respectively. $A_{\mathrm{ex}}$ and $A_{\mathrm{em}}$ are the modified absorbance values post ligand binding to BSA at $\lambda_{\mathrm{ex}}$ and $\lambda_{\mathrm{em}}$, respectively. The fluorescence quenching is broadly divided into two categories, dynamic and static quenching. ${ }^{23}$ Dynamic quenching is due to collisions, and static quenching due to complex formation between the quencher and fluorophore. Several studies have shown that the dynamic quenching constant is expected to increase with a rise in temperature due to increased diffusion rates, whereas a decrease in the static quenching constant is observed with 
increasing temperatures. ${ }^{24,25}$ Therefore, the behavior observed with increased temperatures helped us to conclude the type of quenching involved in this interaction.

\section{SF and 3D fluorescence measurements}

SF spectra for BSA and ROF-BSA complexes were recorded at room temperature. The scanning intervals $\Delta \lambda=15$ and $60 \mathrm{~nm}\left(\Delta \lambda=\lambda_{\text {em }}-\lambda_{\text {ex }}\right)$ were used for the SF measurements. The amino acid Tyr was characterized at $15 \mathrm{~nm}$, whereas $60 \mathrm{~nm}$ characterized the Trp residues. The SF spectral data provided information regarding the conformational changes that might have occurred in BSA after interaction with ROF.

3D-fluorescence spectra were also recorded, which aided in concluding any changes in the BSA conformation postinteraction with ROF.

\section{UV absorption measurements}

Shimadzu UV-1800 spectrophotometer was used to carry out UV-vis absorbance spectral measurements. The spectra for BSA and BSA-ROF complexes using the UV spectrophotometer were recorded at room temperature. The concentration of BSA in the samples was maintained constant for all measurements.

\section{Molecular docking}

Docking studies were carried out to understand the ROFBSA interaction. The MOE-2014 (Molecular Operating Environment), a cocrystallized structure of BSA in complex with naproxen; pdb Code No 4OR0, acquired from Protein Data Bank, was used for docking studies. BSA exists as homodimer of two chains A and B. For the molecular simulation study, we selected chain B of the homodimer. The ligands and the protein receptors were prepared by protonation and the energy minimization using default force field parameters (MMFF94X). Default values (Triangle Matcher) were selected for the remaining docking parameters. London $\mathrm{dG}$ and GBVI/WSA $\mathrm{dG}$ were used for rescoring function 1 and 2, respectively. The binding site on BSA for ROF was selected as per the results obtained from the site probe experiments.

\section{Results and discussion BSA fluorescence quenching}

The interaction between the ligands and the BSA leads to fluorescence quenching. The fluorescence spectrum obtained post the interaction of BSA and ROF is presented in Figure 2.

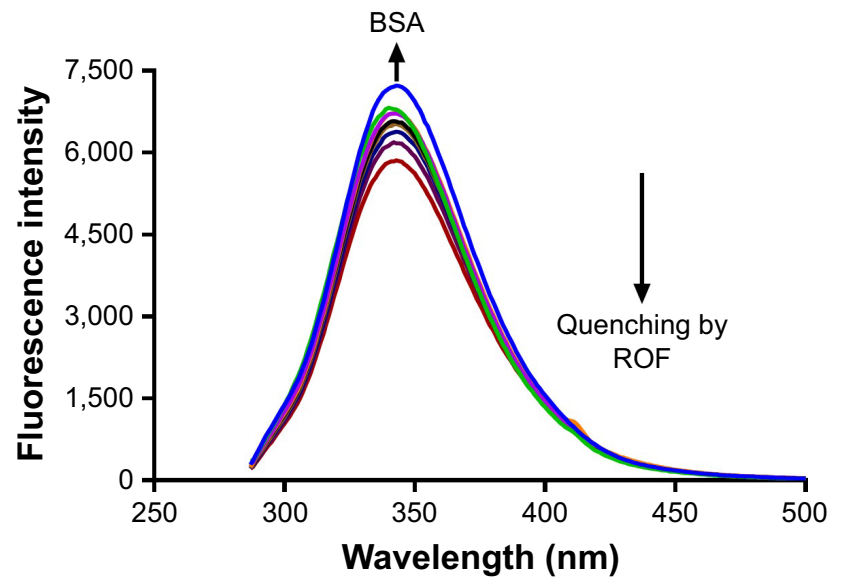

Figure 2 Fluorescence spectra of BSA $(\mathrm{I} .5 \mu \mathrm{M})$ in the presence of ROF at $298 \mathrm{~K}$; ROF concentration $\left(7.46 \times 10^{-7}, 1.49 \times 10^{-6}, 2.24 \times 10^{-6}, 2.98 \times 10^{-6}, 4.48 \times 10^{-6}, 5.97 \times 10^{-6}\right.$, $\left.8.95 \times 10^{-6}, 1.19 \times 10^{-5}\right)$.

Abbreviations: BSA, bovine serum albumin; ROF, roflumilast.

In Figure 2, a reduction in fluorescence intensity can be observed with increasing concentrations of ROF, indicating BSA fluorescence quenching by ROF. The Stern-Volmer equation was used to determine quenching constants at different temperatures and understand the quenching mechanism between ROF and BSA. ${ }^{21}$

$$
\frac{F_{0}}{F}=1+K_{\text {sv }}[Q]=1+K_{\mathrm{q}} \tau_{0}[Q]
$$

In the above equation, $F_{0}$ and $F$ are the fluorescence intensities in the absence and presence of ROF, respectively. $K_{\text {sv }}$ (Stern-Volmer constant), [Q] quencher concentration, and $K_{\mathrm{q}}$ the quenching rate constant. $\tau_{0}$ is valued at $10^{-8} \mathrm{~s}$ and represents the average fluorescence lifetime of BSA. As shown in Figure 3A and Table 1, a strong linear relationship between $F / F_{0}$ and $[Q]$ can be observed for the Stern-Volmer plot demonstrating the contribution of a single quenching mechanism.

The value for $K_{\mathrm{sv}}$ and $K_{\mathrm{q}}$ increased with the rise in temperature, indicative of the involvement of dynamic quenching in the ROF-BSA interaction. However, the $K_{\mathrm{q}}$ values attained were more than the maximum rate constant $\left(2 \times 10^{10} \mathrm{~L} \mathrm{M}^{-1} \mathrm{~S}^{-1}\right)$ that can be attained for scattering collision quenching (Table 2). The higher $K_{\mathrm{q}}$ values suggest a complex formation between ROF and BSA. The BSA spectra (UV absorption) helped in further elucidation of the development of a nonfluorescent complex between BSA and ROF. The noninvolvement of dynamic quenching in the quenching process is indicated by the fact that the absorption spectra of BSA showed an increase after interaction with ROF. This increased 

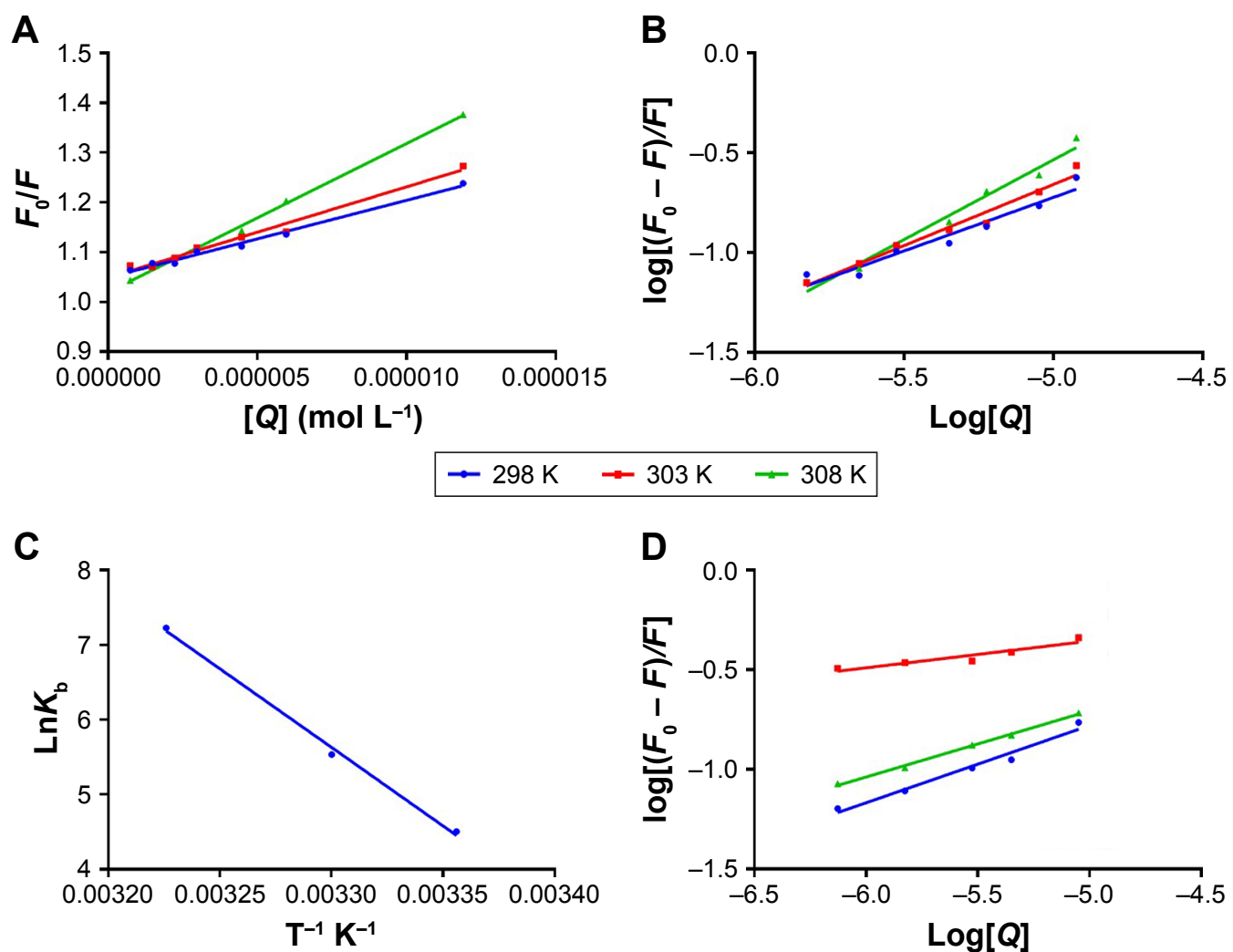

$303 \mathrm{~K}=308 \mathrm{~K}$

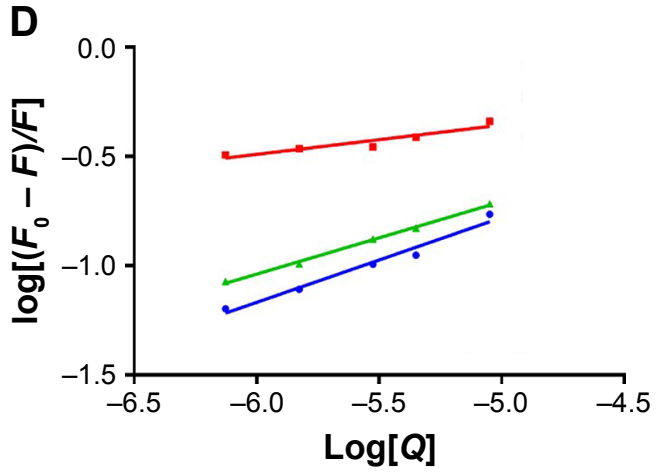

$\rightarrow$ BSA $\rightarrow$ Phenylbutazone $\leftarrow$ Ibuprofen

Figure 3 The plots for interaction between BSA and ROF at different temperatures $(\mathbf{A})$ Stern-Volmer plot, $(\mathbf{B})$ binding constant log $\left[\left(F_{0}-F\right) / F\right]$ vs log $[Q],(\mathbf{C})$ van't Hoff plot, (D) binding constant in presence of site markers at $298 \mathrm{~K}$.

Abbreviations: BSA, bovine serum albumin; ROF, roflumilast.

absorption was possibly because of a complex formation between ROF and BSA, whereas in dynamic quenching the excited state of quencher is liable to the quenching process. As is shown in Figure 4, the UV absorption spectra of BSA increased after addition of ROF, indicating the formation of a nonfluorescent complex (static quenching) ${ }^{23}$

\section{Binding constants of ROF with BSA}

The binding constant $\left(K_{\mathrm{b}}\right)$ was determined for the ROF-BSA complex to obtain information regarding the binding between

Table I $K_{s v}$ and $K_{q}$ values for ROF and BSA at different temperatures

\begin{tabular}{lll}
\hline$T(K)$ & $\begin{array}{l}K_{\mathrm{sv}} \pm \mathbf{S D} \times 1 \mathbf{0}^{4} \\
\left(\mathbf{L ~ m o l} \mathbf{~ m}^{-1}\right)\end{array}$ & $\begin{array}{l}\boldsymbol{K}_{\mathrm{q}} \times \mathbf{l 0}^{12} \\
\left(\mathrm{~L} \mathrm{~mol}^{-1} \mathbf{~ s}^{-1}\right)\end{array}$ \\
\hline 298 & $1.49 \pm 0.11$ & 1.49 \\
303 & $1.78 \pm 0.14$ & 1.78 \\
308 & $2.82 \pm 0.23$ & 2.82 \\
\hline
\end{tabular}

Abbreviations: BSA, bovine serum albumin; $K_{\mathrm{q}}$, quenching rate constant; $K_{\mathrm{sv}}$, Stern-Volmer constant; ROF, roflumilast.
BSA and ROF. The binding constant and number of binding sites $(n)$ were determined using the equation: ${ }^{24}$

$$
\log \frac{\left(F_{0}-F\right)}{F}=\log K_{\mathrm{b}}+n \log [Q]
$$

A plot between $\log \left[\left(F_{0}-F\right) / F\right]$ versus $\log [Q]$ (Figure 3B) was used calculate the $K_{\mathrm{b}}$ and $n$ values, are presented in Table 2. Over the studied range of temperature, the number of binding sites $(n)$ was unity, suggesting that only one type of binding site was present on BSA that could bind to ROF. A higher $K_{\mathrm{b}}$ value indicates a stronger binding interaction between the protein and the ligand. The $K_{\mathrm{b}}$ values showed an increase in binding strength with increasing temperatures in interaction of ROF and BSA.

\section{SF spectra}

$\Delta \lambda$ values of 15 or $60 \mathrm{~nm}$ were used to record the SF spectra of both BSA and BSA-ROF complex. These spectra provided characteristic evidence about the microenvironment 
Table 2 ROF-BSA interaction binding constant and thermodynamic values at different temperatures

\begin{tabular}{|c|c|c|c|c|c|c|}
\hline$T(K)$ & $\log K_{b} \pm S D$ & $K_{\mathrm{b}} \pm \mathrm{SD}\left(\mathrm{L} \mathrm{mol}^{-1}\right)$ & $n$ & $\left.\Delta G(\mathbf{k J ~ m o l})^{-1}\right)$ & $\Delta H\left(\mathrm{~kJ} \mathrm{~mol} \mathrm{~m}^{-1}\right)$ & $\Delta S\left(\mathrm{~J} \mathrm{~mol}^{-1} \mathrm{~K}^{-1}\right)$ \\
\hline 298 & 1.96 & $0.09 \times 10^{2} \pm 0.04$ & 0.54 & -11.04 & 175 & 624.2 \\
\hline 303 & 2.40 & $2.53 \times 10^{2} \pm 0.15$ & 0.61 & -14.17 & & \\
\hline 308 & 3.37 & $1.38 \times 10^{3} \pm 0.29$ & 0.80 & -18.54 & & \\
\hline
\end{tabular}

Abbreviations: $B S A$, bovine serum albumin; ROF, roflumilast; $K_{b}$, binding constant.

of Tyr and Trp residues of BSA. ${ }^{13}$ A red shift in the SF spectra indicates a decreased hydrophobicity around the Tyr and Trp residues and increased stretching capacity of the peptide chain. A blue shift in the emission spectra indicates increased hydrophobicity in the Tyr and Trp microenvironments and increased folding capacity for the biomolecule. The spectra (Figure 5) clearly show a decrease in the fluorescence intensity after ROF addition. There was shift of $1 \mathrm{~nm}$ (red shift) at both $\Delta \lambda=15$ and $60 \mathrm{~nm}$ specifying certain microenvironmental changes for Tyr and Trp upon binding to ROF.

\section{Three-dimensional fluorescence spectroscopy}

The three-dimensional fluorescence spectral analyses of BSA and BSA-ROF are presented in Figure 6. Two emission peaks were observed for the analyzed BSA solutions denoted by Peak I (Rayleigh scattering peak) and Peak II (characterizes Trp and Tyr residues of BSA). ${ }^{6,9}$ The intensity and positioning of Peak II are dependent on the microenvironment of Tyr and Trp residues, and any change in this microenvironment can induce some sort

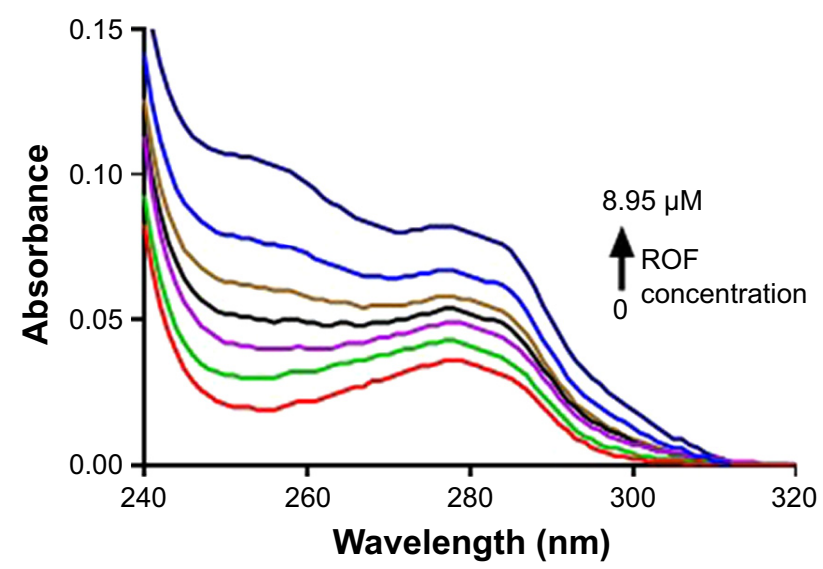

Figure 4 Spectra for UV absorption of BSA $(1.5 \mu \mathrm{M})$ and BSA in presence of ROF at room temperature.

Note: ROF concentrations used were $7.46 \times 10^{-7}, 1.49 \times 10^{-6}, 2.98 \times 10^{-6}, 4.48 \times 10^{-6}$, $5.97 \times 10^{-6}$, and $8.95 \times 10^{-6}$.

Abbreviations: BSA, bovine serum albumin; ROF, roflumilast. of conformational change in BSA. The Peak I in the BSA is formed as a result of $\pi-\pi^{*}$ transition of polypeptide structures and is also related to the particle size of the protein. There was decrease in the intensity of Peak II of BSA suggesting fluorescence quenching by ROF, and thus the change in the microenvironmental vicinity might have caused conformational changes in BSA. Moreover, a decrease in the Peak I suggests that ROF addition to BSA might have decreased its diameter, reflecting a conformational change in BSA.

\section{Thermodynamic parameters and binding model}

The major intramolecular interaction forces that are involved in binding proteins to drug ligand include the hydrogen bond, van der Waals forces, and hydrophobic interactions. Thermodynamic parameters including Gibbs free energy change $\left(\Delta G^{0}\right)$, enthalpy change $\left(\Delta H^{0}\right)$, and entropy change $\left(\Delta S^{0}\right)$ help in establishing the type of forces involved in the binding interaction. ${ }^{26} \Delta H^{0}$ and $\Delta S^{0}<0$ specify contributions of van der Waals force and/or hydrogen bonding, whereas $\Delta H^{0}>0$ and $\Delta S^{0}>0$ specify a hydrophobic interaction. In case, where $\Delta H^{0} \sim 0$ and $\Delta S^{0}>0$, the involvement of electrostatic force is expected. Similarly, $\Delta G^{0}<0$ specified a spontaneous binding process.

Hydrophobic interaction between BSA and ROF was suggested on the basis of positive values of $\Delta H^{0}$ and $\Delta S^{0}$. The negative $\Delta G^{0}$ specified a spontaneous binding process between ROF and BSA (Table 2). Figure $3 \mathrm{C}$ represents the van't Hoff plot for BSA-ROF interaction.

\section{Binding site identification of ROF}

The site for binding of ROF to BSA was identified using site competition. Phenylbutazone and ibuprofen (Figure 7), which act as markers for Site I and Site II, respectively, were used the identification of the binding site in this study..$^{27}$ The fluorescence spectra were recorded for BSA site marker complexes and ROF. Binding constants for the ROF with BSA were calculated in the presence of site markers using 

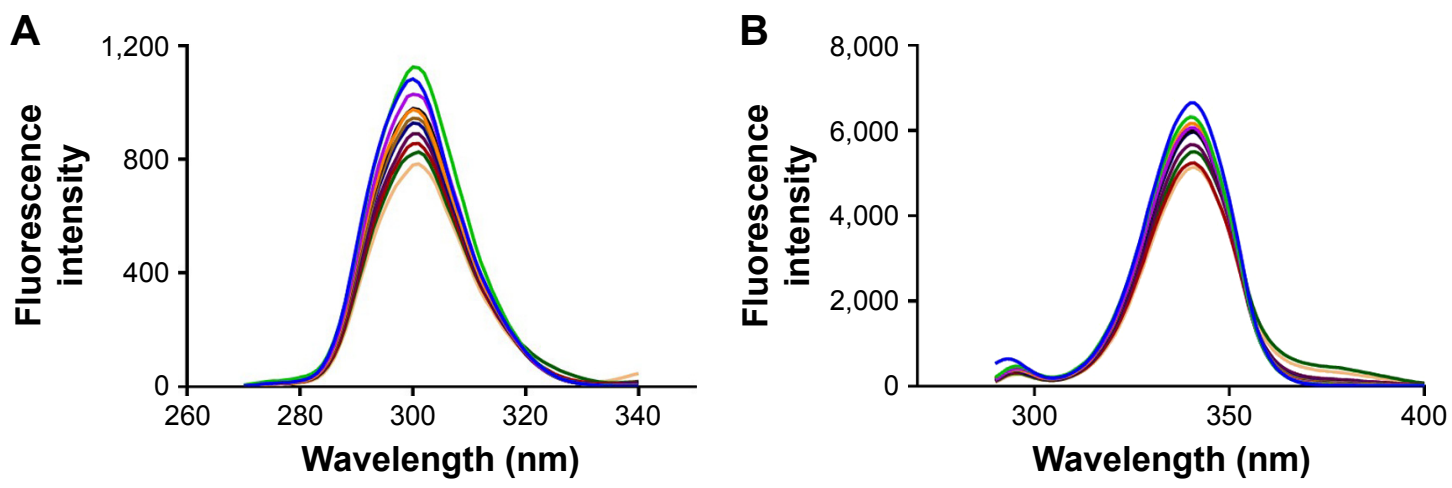

Figure 5 BSA $(1.5 \mu \mathrm{M})$ and ROF $\left(7.46 \times 10^{-7}, 1.49 \times 10^{-6}, 2.24 \times 10^{-6}, 2.98 \times 10^{-6}, 4.48 \times 10^{-6}, 5.97 \times 10^{-6}, 8.95 \times 10^{-6}, 1.19 \times 10^{-5}, 1.49 \times 10^{-5}\right.$, and $\left.1.64 \times 10^{-5}\right) \mathrm{SF}$ spectra $(\mathbf{A}) \Delta \lambda=15 \mathrm{~nm}$; (B) $\Delta \lambda=60 \mathrm{~nm}$ at room temperature.

Abbreviations: BSA, bovine serum albumin; ROF, roflumilast; SF, synchronous fluorescence.

double logarithmic plots of $\log \left[\left(F_{0}-F\right) / F\right]$ versus $\log [\mathrm{Q}]$ (Figure 3D). The binding constant $\left(K_{\mathrm{b}}\right)$ of BSA with ROF decreased significantly in the presence of phenylbutazone compared to $K_{\mathrm{b}}$ in absence of phenylbutazone. A slight

A

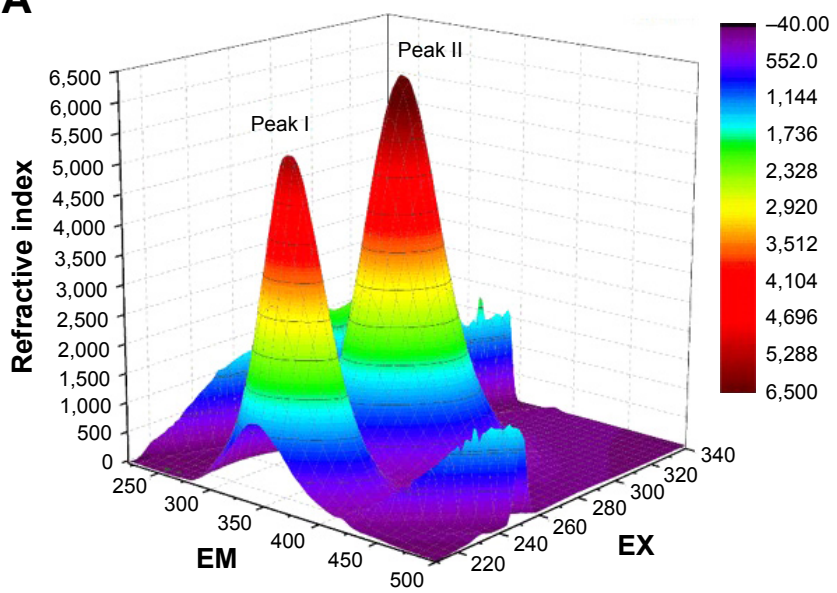

C

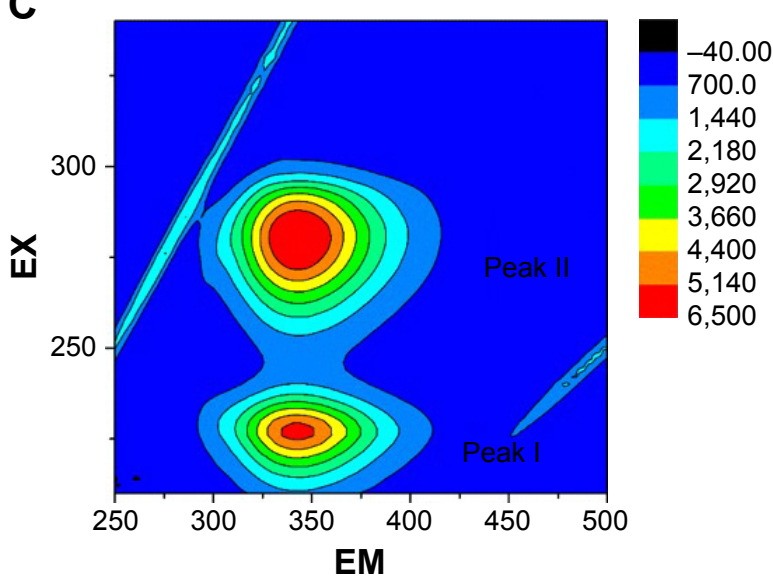

change was observed in the $K_{\mathrm{b}}$ of BSA with ROF in the presence of ibuprofen, indicating a competition between ROF and phenylbutazone for binding to BSA Site I. A molecular simulation program (MOE) was used for the simulation
B
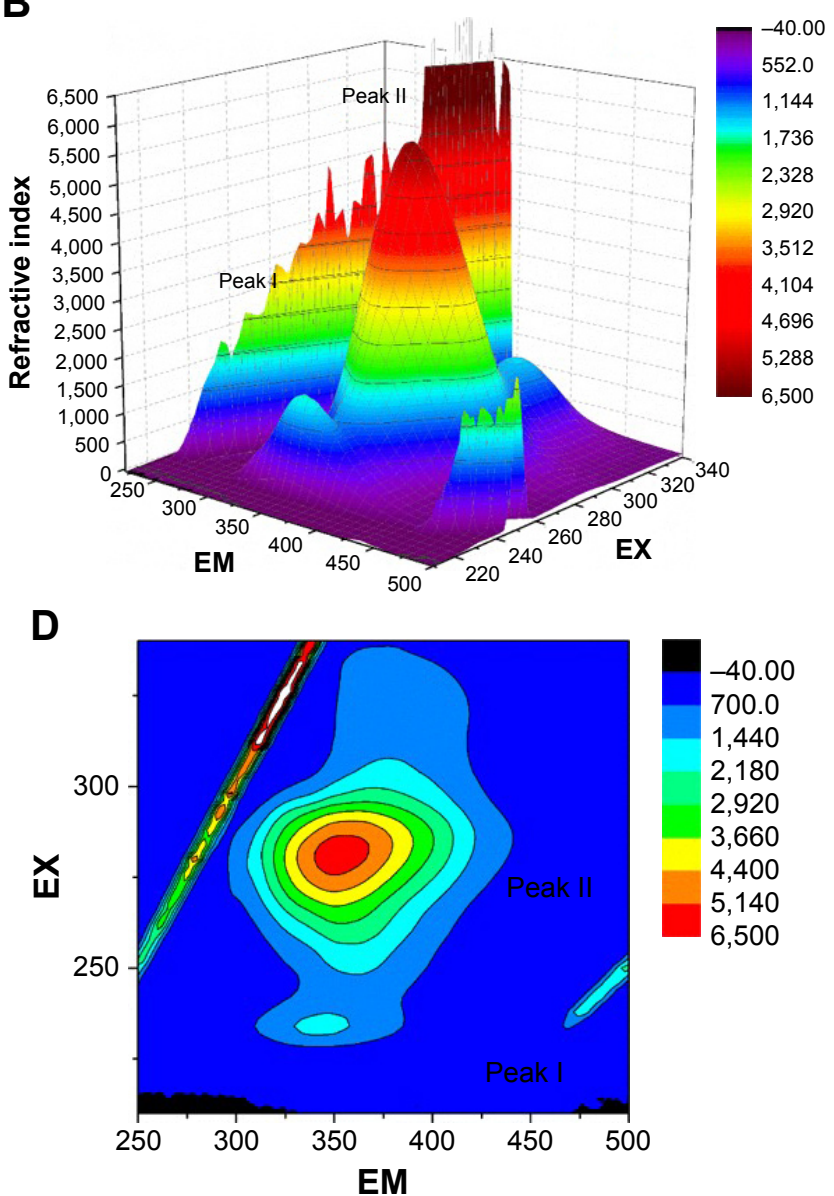

Figure 6 Three-dimensional fluorescence analysis, BSA (A and C) BSA-ROF complex (B and D) represent the contour plot. Abbreviations: BSA, bovine serum albumin; ROF, roflumilast. 
A

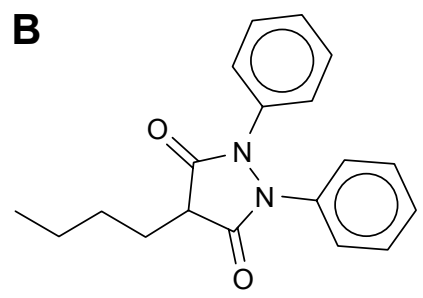

Figure $\mathbf{7}$ Chemical structure of $(\mathbf{A})$ ibuprofen; $(\mathbf{B})$ phenylbutazone.

of ROF and BSA interactions. The docking experiments (Figure 8A) further revealed that ROF binds to Site I (subdomain IIA) of BSA within a hydrophobic cavity. ${ }^{28}$ The docking results were supported by the binding site probe experimental results. Both the experiments showed that phenylbutazone and ROF bind to the same site on BSA, inferring Site I (subdomain IIA) hydrophobic pocket of BSA as the binding site for ROF. The free energy change $\left(\Delta G^{0}\right)$ calculated from the molecular docking simulation was determined to be $-6.43 \mathrm{kcal} \mathrm{mol}^{-1}$ and was lower than the $\Delta G^{0}$ found experimentally $\left(3.39 \mathrm{kcal} \mathrm{mol}^{-1}\right)$.

The binding mode study further revealed that ROF interacts with the BSA subdomain IIA hydrophobic region. The hydrophobic amino acids (Val-342, Ala-209, Leu-210, Leu-480, Leu-197, Leu-346, Leu-454), polar residues (Trp-213, Ser-201, Ser-343, Ser-453), and charged residues (Arg-217, Arg-198, Arg-194, Asp-450) surround the embedded ROF. The amino acid residues Arg-194 and
Ser-343 formed hydrogen bonds between ROF and BSA (Figure 8B).

\section{BSA conformational changes}

The conformational changes in BSA after interaction with ROF were investigated with UV absorption spectroscopy. The absorption spectra for BSA and BSA-ROF are presented in Figure 4. An increase in the absorption intensity was observed with gradual addition of ROF. The intensity of the peak at $280 \mathrm{~nm}$ is because of $\pi-\pi^{*}$ transition of aromatic amino acids (Trp, Tyr, and Phe). A complex formation between BSA and ROF is further suggested based on the fact that a blue shift was also observed for the $\lambda_{\max }$ of BSA. ${ }^{29}$

\section{Conclusion}

The BSA and ROF binding interaction was explored in this study using different methodologies at the molecular structural level. ROF quenched the fluorescence of BSA by forming a complex with BSA. ROF was also found to bind with BSA at subdomain IIA (Site I) leading to conformational changes in BSA. The contribution of hydrophobic forces in the binding process of ROF with BSA was indicated by the positive enthalpy and entropy values. The UV-vis and SF spectroscopic data indicated that the interaction between ROF and BSA caused alterations in the BSA conformation. Our study will help in further investigation of the pharmacological behavior of ROF.
A

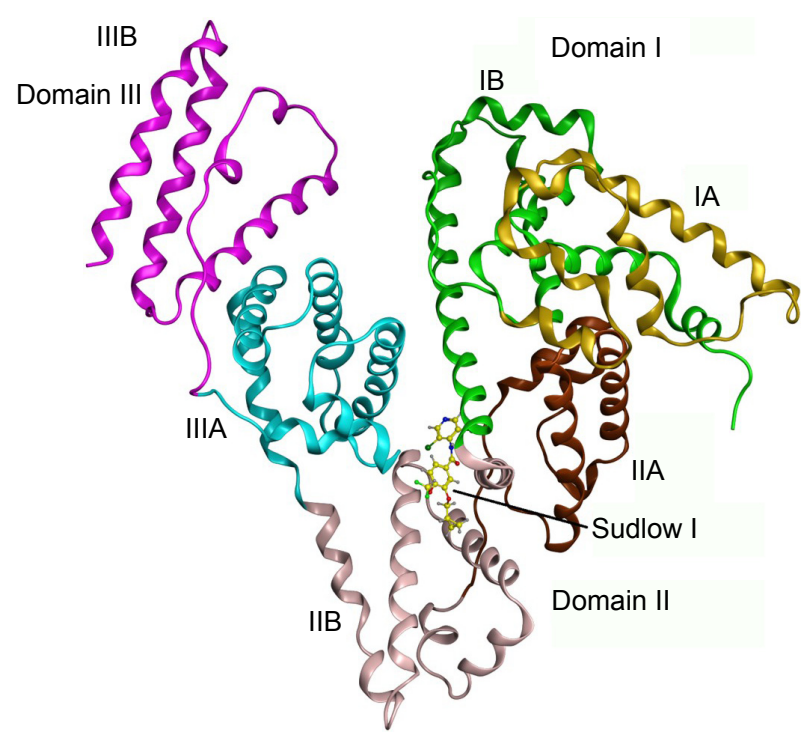

B

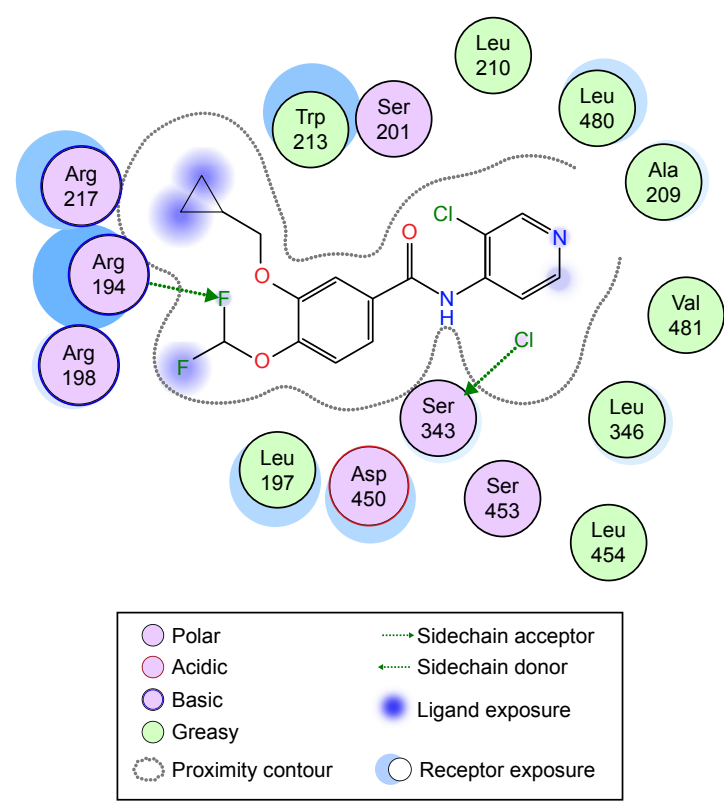

Figure 8 (A) Three-dimensional representation of the interaction of ROF with BSA, (B) a two-dimensional representation of amino acids that surround ROF. Abbreviations: BSA, bovine serum albumin; ROF, roflumilast. 


\section{Acknowledgment}

The authors extend their sincere appreciation to the Deanship of Scientific Research, King Saud University, for funding the research group no. RG-1435-073.

\section{Disclosure}

The authors report no conflicts of interest in this work.

\section{References}

1. Halbert R, Natoli J, Gano A, Badamgarav E, Buist AS, Mannino D. Global burden of COPD: systematic review and meta-analysis. Eur Respir J. 2006;28(3):523-532.

2. Wedzicha JA, Calverley PM, Rabe KF. Roflumilast: a review of its use in the treatment of COPD. Int J Chron Obstruct Pulmon Dis. 2016;11: $81-90$.

3. Garnock-Jones KP. Roflumilast: a review in COPD. Drugs. 2015;75(14): $1645-1656$.

4. Boswell-Smith V, Spina D. PDE4 inhibitors as potential therapeutic agents in the treatment of COPD-focus on roflumilast. Int $J$ Chron Obstruct Pulmon Dis. 2007;2(2):121-129.

5. Sułkowska A. Interaction of drugs with bovine and human serum albumin. J Mol Struct. 2002;614(1-3):227-232.

6. Shi JH, Pan DQ, Jiang M, Liu TT, Wang Q. Binding interaction of ramipril with bovine serum albumin (BSA): insights from multispectroscopy and molecular docking methods. J Photochem Photobiol. 2016;164:103-111.

7. Shi JH, Pan DQ, Wang XX, Liu TT, Jiang M, Wang Q. Characterizing the binding interaction between antimalarial artemether (AMT) and bovine serum albumin (BSA): Spectroscopic and molecular docking methods. J Photochem Photobiol. 2016;162:14-23.

8. Karthikeyan S, Bharanidharan G, Kesherwani M, et al. Insights into the binding of thiosemicarbazone derivatives with human serum albumin: spectroscopy and molecular modelling studies. J Biomol Struct Dyn. 2016;34(6):1264-1281.

9. Wani TA, AlRabiah H, Bakheit AH, Kalam MA, Zargar S. Study of binding interaction of rivaroxaban with bovine serum albumin using multi-spectroscopic and molecular docking approach. Chem Cent J. 2017;11(1):134.

10. Suryawanshi VD, Walekar LS, Gore AH, Anbhule PV, Kolekar GB. Spectroscopic analysis on the binding interaction of biologically active pyrimidine derivative with bovine serum albumin. J Pharm Anal. 2016;6(1):56-63.

11. Wani TA, Bakheit AH, Al-Majed AA, Bhat MA, Zargar S. Study of the interactions of bovine serum albumin with the new anti-inflammatory agent 4-(1, 3-dioxo-1, 3-dihydro-2 $H$-isoindol-2-yl)-N'-([4-ethoxyphenyl] methylidene) benzohydrazide using a multi-spectroscopic approach and molecular docking. Molecules. 2017;22(8):1258.

12. Steinhardt J, Krijn J, Leidy JG. Differences between bovine and human serum albumins. Binding isotherms, optical rotatory dispersion, viscosity, hydrogen ion titration, and fluorescence effects. Biochemistry. 1971;10(22):4005-4015.
13. Majorek KA, Porebski PJ, Dayal A, et al. Structural and immunologic characterization of bovine, horse, and rabbit serum albumins. Mol Immunol. 2012;52(3-4):174-182.

14. Wani TA, Bakheit AH, Abounassif M, Zargar S. Study of interactions of an anticancer drug neratinib with bovine serum albumin: spectroscopic and molecular docking approach. Front Chem. 2018;6:47.

15. Wani TA, Bakheit AH, Zargar S, Hamidaddin MA, Darwish IA. Spectrophotometric and molecular modelling studies on in vitro interaction of tyrosine kinase inhibitor linifanib with bovine serum albumin. PLoS One. 2017;12(4):e0176015.

16. Shahabadi N, Fili SM. Molecular modeling and multispectroscopic studies of the interaction of mesalamine with bovine serum albumin. Spectrochim Acta A Mol Biomol Spectrosc. 2014;118:422-429.

17. Jahanban-Esfahlan A, Panahi-Azar V, Sajedi S. Spectroscopic and molecular docking studies on the interaction between $\mathrm{N}$-acetyl cysteine and bovine serum albumin. Biopolymers. 2015;103(11):638-645.

18. Kandagal P, Ashoka S, Seetharamappa J, Shaikh S, Jadegoud Y, Ijare O. Study of the interaction of an anticancer drug with human and bovine serum albumin: spectroscopic approach. J Pharm Biomed Anal. 2006; 41(2):393-399.

19. Khorsand Ahmadi S, Mahmoodian Moghadam M, Mokaberi P, Reza Saberi M, Chamani J. A comparison study of the interaction between $\beta$-lactoglobulin and retinol at two different conditions: spectroscopic and molecular modeling approaches. J Biomol Struct Dyn. 2015;33(9):1880-1898.

20. Chamani J, Vahedian-Movahed H, Saberi MR. Lomefloxacin promotes the interaction between human serum albumin and transferrin: a mechanistic insight into the emergence of antibiotic's side effects. J Pharm Biomed Anal. 2011;55(1):114-124.

21. Sattar Z, Saberi MR, Chamani J. Determination of LMF binding site on a HSA-PPIX complex in the presence of human holo transferrin from the viewpoint of drug loading on proteins. PLoS One. 2014;9(1):e84045.

22. Lakowicz JR. Introduction to fluorescence. Principles of Fluorescence Spectroscopy. Berlin: Springer; 1999:1-23.

23. Grigoryan K, Ghazaryan A. Quenching mechanism of human serum albumin fluorescence by gangleron. Chem Biol. 2013;2:6-10.

24. Shahabadi N, Hadidi S, Feizi F. Study on the interaction of antiviral drug "Tenofovir" with human serum albumin by spectral and molecular modeling methods. Spectrochim Acta A Mol Biomol Spectrosc. 2015;138: 169-175.

25. Min J, Meng-Xia X, Dong Z, Yuan L, Xiao-Yu L, Xing C. Spectroscopic studies on the interaction of cinnamic acid and its hydroxyl derivatives with human serum albumin. J Mol Struct. 2004;692(1-3):71-80.

26. Ross PD, Subramanian S. Thermodynamics of protein association reactions: forces contributing to stability. Biochemistry. 1981;20(11): 3096-3102.

27. Zhang Q, Ni Y, Kokot S. Molecular spectroscopic studies on the interaction between ractopamine and bovine serum albumin. J Pharm Biomed Anal. 2010;52(2):280-288.

28. Sudlow G, Birkett D, Wade D. Further characterization of specific drug binding sites on human serum albumin. Mol Pharmacol. 1976;12(6): 1052-1061.

29. Cui FL, Fan J, Li JP, Hu ZD. Interactions between 1-benzoyl-4-pchlorophenyl thiosemicarbazide and serum albumin: investigation by fluorescence spectroscopy. Bioorg Med Chem. 2004;12(1):151-157.

\section{Publish your work in this journal}

Drug Design, Development and Therapy is an international, peerreviewed open-access journal that spans the spectrum of drug design and development through to clinical applications. Clinical outcomes, patient safety, and programs for the development and effective, safe, and sustained use of medicines are the features of the journal, which

\section{Dovepress}

has also been accepted for indexing on PubMed Central. The manuscript management system is completely online and includes a very quick and fair peer-review system, which is all easy to use. Visit http://www.dovepress.com/testimonials.php to read real quotes from published authors. 